\title{
cosmetics
}

ISSN 2079-9284

www.mdpi.com/journal/cosmetics

Review

\section{New Trends in Cosmetics: By-Products of Plant Origin and Their Potential Use as Cosmetic Active Ingredients}

\author{
Ani Barbulova ${ }^{1}$, Gabriella Colucci ${ }^{2}$ and Fabio Apone ${ }^{2, *}$ \\ 1 Arterra Bioscience srl, via B. Brin 69, 80142 Napoli, Italy; E-Mail: ani@arterrabio.it \\ 2 Vitalab srl/Arterra Bioscience srl, via B. Brin 69, 80142 Napoli, Italy; \\ E-Mail: gcolucci@arterrabio.it \\ * Author to whom correspondence should be addressed; E-Mail: fapone@arterrabio.it; \\ Tel.: +39-081-658-4411; Fax: +39-081-214-4864.
}

Academic Editor: Carla Villa

Received: 13 February 2015 / Accepted: 10 April 2015 / Published: 16 April 2015

\begin{abstract}
In recent years, the amount of waste deriving from industrial processes has increased substantially. Many industries produce different types of disposable by-products, rich in valuable compounds. Their characterization and valorization could not only convert them into high value products with application in diverse biotechnological fields, such as Pharmaceutics, Food or Cosmetics, but would also reduce the waste environmental impact and the related treatment costs. There are many examples of cosmetic active ingredients deriving from fish, meat and dairy products, but in the present review we would like to focus on the potentialities and the current use of compounds and extracts deriving from agronomical disposable wastes in the cosmetic field. These types of products are effective, inexpensive and bio-sustainable, and thus represent a valid alternative to the regular plant derived extracts, more commonly adopted in cosmetic formulations. Moreover, if the waste products come from organic farming, they are certainly an even more valuable source of safe extracts for Cosmetics, since they lack any residual pesticide or potentially toxic chemical.
\end{abstract}

Keywords: plant; by-products; Cosmetics 


\section{Introduction}

Fruit and vegetables represent an essential part of our diet and their undeniable nutritional value has been intensively studied for decades. In addition to "primary" metabolites (e.g., saccharides, lipids and amino acids), the plants synthesize a wide variety of "secondary" metabolites, which have fundamental roles in plant protection against both biotic and abiotic stresses [1]. Moreover, secondary metabolites mediate many types of interactions with the environment and with other organisms that occupy the same ecological habitat [2]. For centuries, they have been of great interest for humans as flavors, fragrances, dyes, preservatives and pharmaceuticals as well: in fact, epidemiological studies indicated that the consumption of fruit and vegetables has brought huge health benefits, such as reduced risk for coronary heart disease and stroke, as well as certain types of cancer [3]. This protective action was mainly attributed to the effects of the dietary fibers and the secondary metabolites, mainly carotenoids and flavonoids with strong antioxidant activity [4]. The most abundant carotenoid, beta-carotene, contained in red and orange fruits such as tomatoes, was capable of neutralizing the free radicals and increasing the cellular antioxidant defenses [3]. Among the flavonoids, the most important examples of compounds with therapeutic activity are: the flavanols, as catechins, epicatechins and procyanidins, present in apples and grapes, that contribute to maintain the heart health; the flavanones, the dominant flavonoid class in citrus fruits; the anthocyanidins, mostly present in berries, cherries and red grapes, that increase the antioxidant defenses and enhance the brain functions [3].

Thanks to all these properties and to the intensive campaign to promote the benefits of fresh green products, the consumption of fruit and vegetables has increased substantially in the last decades and the amounts of wastes and residues, produced by agro-food industries, are now estimated to be around 800,000 tons per year of fresh matter globally, without considering the wastage during the food processing [5]. Nowadays most agricultural waste is processed using basic waste management and valorization strategies as animal feed, production of composts and fertilizer, as well as anaerobically digestion to biogas. Since this type of processing is associated with high costs of drying, storage and transport, an efficient and environmentally friendly utilization and recycling of this waste is becoming more and more important [6].

The agricultural processing industries generate from $10 \%$ to $60 \%$ of raw materials as solid waste and it was proven that in some cases the products from wastes were even more valuable than the main products [7]. Table 1 illustrates the waste materials generated from some fruit and vegetable processing industries, expressed as percentages, which we prefer to call "by-products" to underline their large potentialities to be recycled as valuable products for industry. These by-products are made up mainly of skins, seeds, stems, leaves, waste waters and unusable pulp which are normally discarded and in some cases represent more than $40 \%$ of total plant food (such as the cases of artichoke, asparagus, mango, citrus fruits, papaya, pineapple) [7].

It is well known that the food by-products are still very rich of sugars, minerals, organic acids, dietary fiber and bioactive compounds, as polyphenols and carotenoids, analogously to their edible counterparts. Their appealing composition, together with the growing interest in finding natural ingredients as an alternative to synthetic substances, has determined the food by-products an economically attractive source for the production of high value compounds in different industrial fields, including Food, Nutraceutics, Pharmaceutics and Cosmetics [8]. This review has the scope to 
provide some examples among the most important applications of agricultural food by-products in Cosmetics, and discuss their performance as efficacy and safety.

Table 1. By-product quantities generated from some fruit and vegetable processing industries [7].

\begin{tabular}{ccc}
\hline Plant & By-Products & Edible Part \\
\hline Agave & $40 \%$ (rind and pith) & $60 \%$ \\
Apple & $11 \%$ (pulp and seed core) & $89 \%$ \\
Artichoke & $60 \%$ (outer bracts, receptacles and stems) & $40 \%$ \\
Asparagus & Up to $40 \%-50 \%$ (spear) & $50 \%-60 \%$ \\
Banana & Up to 30\% (peel) & $70 \%$ \\
Cactus cladodes & $20 \%$ (spines, glochids and peel) & $80 \%$ \\
Carrot & $30 \%-40 \%$ (pomace) & $60 \%-70 \%$ \\
Mandarin & $16 \%$ (peels) & $84 \%$ \\
Mango & $42 \%$ (seeds, peels, unusable pulp) & $58 \%$ \\
Citrus fruits & $66 \%$ (peel) & $44 \%$ \\
Papaya & $47 \%$ (seeds, peels, unusable pulp) & $53 \%$ \\
Passion fruit & $75 \%$ (rind and seeds) & $25 \%$ \\
Pineapple & $52 \%$ (core, peels, top, pulp) & $48 \%$ \\
Potato & $15 \%-40 \%$ (peel) & $60 \%-85 \%$ \\
Tomato & $3 \%-7 \%$ (peel and seeds) & $93 \%-97 \%$ \\
\hline
\end{tabular}

\section{Citrus Fruit Processing By-Products}

Citrus plants (genus Citrus in family Rutaceae), also known as agrumes, are one of the world's major fruit crops with global availability and popularity contributing to human diet. Fresh citrus fruits are a source of dietary fibers, associated with gastrointestinal disease prevention and lowered circulating cholesterol; they are rich in vitamin C, B vitamins (thiamin, pyridoxine, niacin, riboflavin, pantothenic acid, and folate) and phytochemicals, such as carotenoids, flavonoids, and limonoids [9]. These biological constituents are of crucial importance in human health improvement due to their antioxidant properties, ability to be converted to vitamin A (for example the $\beta$-cryptoxanthin), and to protect from various chronic diseases. Although many citrus fruits, such as oranges, tangerines, and grapefruits can be eaten fresh, about a third of citrus fruit worldwide is utilized after processing, and orange/lemon juice production accounts for nearly $85 \%$ of total processed consumption [9].

Citrus processing generates high quantity of agricultural waste, and most of it is in the form of press-cakes, that are pricy to dispose due to the costs for handling and transport. In order to give value to this waste, an investigation was carried out into the anti-melanogenic effects of ethanol extracts of the press cakes of Citrus unshiu, which were mainly grown in Jeju Island in Korea and the fruit peel was extensively used in the traditional medicine as digestive or to treat severe and atopic dermatitis [10,11].

The development of novel whitening phytochemicals from natural sources has become a very popular trend. Melanin, the end product of melanogenesis, determines the color of human skin, hair, and eyes, and although its photoprotective role in absorbing free radicals in the cells and shielding from UV light, its overproduction and accumulation in the skin may cause skin disorders and aesthetic problems, such as hyper-pigmentation and dark spots [12,13]. 
Melanin production is determined by the activity of a key enzyme, tyrosinase, and its transcription factor MITF (Microphthalmia-associated transcription factor); thus, a down-regulation of tyrosinase activity or expression would be responsible for a reduced melanin content in the skin [10]. In addition, tyrosinase-related protein TRP-1 and TRP-2 are also important for melanin synthesis and are regulated by MITF [14].

Citrus press cakes composed of C. unshiu fruit peels were extracted by using ethanol as solvent and the extract tested for its capacity to inhibit melanin content, tyrosinase activity and the protein expression profile of TRP-1, TRP-2 and MITF in murine B16F10 melanocytes [10]. It was established that the treatment with citrus press cake extracts significantly reduced the cellular melanin content, through inhibition of the tyrosinase activity and the transcription factors TRP-1 and TRP-2 in a dose dependent manner. Based on a Western blot analysis (analytical technique, used to detect specific proteins in a sample), the upstream transcription factor MITF was also down-regulated in a dose-dependent manner. These results, which should be complemented with further in vivo assessment of the biological activity of citrus press cake extracts, suggested that this type of food by-product was a promising candidate for treating skin pigmentation disorders [10].

Besides the ethanol extracts, citrus by-products are important sources of essential oils [15]. Essential oils obtained from plants are well known due to their strong antimicrobial, antioxidant and anti-inflammatory properties and have a number of potential applications, including use as food additives, preservatives against spoilage, pharmaceuticals and cosmeceuticals [16]. Thus, an investigation was carried out into the potential use of an essential oil, obtained from C. unshiu peel wastes using hydro-distillation, as anti-inflammatory and anti-microbial agent in Cosmetics [17]. The chemical analysis of this oil revealed the presence of six major compounds (comprising up to $94.5 \%$ of the total oil composition): limonene (80.5\%), $\gamma$-terpinene $(6.80 \%)$, cymene $(4.02 \%), \beta$-myrcene $(1.59 \%)$, $\alpha$-pinene $(1.02 \%)$ and $\alpha$-terpinolene $(0.56 \%)$ [15]. The oil showed a strong antibacterial activity due to its monoterpen composition that destroyed the cellular integrity of the microbial cells, inhibiting the respiration process. It was also demonstrated that this oil was able to down-regulate nitric oxide (NO) synthesis. NO is an endogenous free radical species, synthesized in various animal cell and tissues by nitric oxide synthase (NOS), and activates the inflammatory process in the tissues [15]. After exposure to stress inducers, such as a bacterial lipopolysaccharide (LPS), NO production can be induced in various cells, triggering a transduction cascade leading to tissue damage and inflammation [18]. When tested on LPS-treated RAW264.7 cell line (macrophages of the immune system), C. unshiu oil, extracted from the press-cakes, produced a strong inhibition of NO synthesis, suggesting that it could be a valuable anti-inflammatory ingredient to be employed in cosmetic formulas [17].

\section{Tomato and Olive Processing By-Products}

Two of the most important food processes which generate valuable products exported all over the world are those employed by the tomato processing industries and by the olive oil production farms, mostly located in the Mediterranean areas.

On a global scale, the annual production of fresh tomatoes amounts to approximately 160 million tons and almost 40 million tons of tomatoes are processed as tomato products: tomato paste, peeled or unpeeled, whole or chopped tomatoes [19]. Tomato processing produces high amounts of interesting 
by-products, peels, pulps and seeds, also called "tomato pomace", that are extremely rich in anti-oxidant compounds: in particular the lycopene, a compound known for its role in disease prevention, is in high demand from food, pharmaceutical and cosmetic industries [20].

Commercially available lycopene mostly comes from standardized tomato fruit extraction or from chemical synthesis, but the product is generally expensive due to the complicated extraction procedures. The peel fraction of tomato by-products contains lycopene up to five times more than the pulp (on wet basis), but its high moisture levels and susceptibility to microbial spoilage make the storage and processing of this material problematic [21]. Lycopene extractability by conventional food-grade organic solvents, such as hexane, ethanol and ethyl acetate, is extremely low, at least under the conditions that normally preserve the activity of the carotenoids in vivo [22]. It was established that an enzymatic pre-treatment of tomato peels with a pectanolitic enzyme preparations, together with the use of surfactant-assisted extraction, significantly increased the level of lycopene recovery. In addition, the use of organic solvents for recovery of the lipophilic lycopene was avoided making the process of its extraction environmentally friendly, ensuring a direct lycopene use in food and cosmetics industries [21].

Besides tomatoes, olive (Olea europea) oil is also an important part of the Mediterranean diet associated with an improved health status in terms of lower incidence of coronary heart diseases. It has already been used as a key ingredient in a wide variety of cosmetic products - soaps, face and body creams, lotions, shampoos - due to the presence of monounsaturated fatty acids and phenolic compounds. These compounds are predominantly contained in the aqueous phase of the olives and exert diverse biological activities in endothelial and epithelial cells, platelets, neurons, cells of the immune system, and neoplastic cells [23]. The main phenolic compounds, hydroxytyrosol and oleuropein, and their derivatives, which give extra-virgin olive oil its bitter taste, have powerful antioxidant activity both in vivo and in vitro. There are a number of studies regarding the oleuropein and its pharmacological activities including antioxidant, anti-inflammatory, anti-atherogenic, anti-cancer, antimicrobial, antiviral, hypolipidemic and hypoglycemic effect [24]. Hydroxytyrosol and oleuropein, together with other antioxidant compounds present in the olives, are highly soluble in water, thus only a low percentage dissolves in the lipidic fraction of the olives [25].

During the olive oil extraction process, most of the polyphenols are lost in the Oil Vegetation Waters (OVW), which represent the water-soluble fractions of the olives, which are separated from the oil. According with the figures of International Olive Council, the worldwide consumption of olive oil increased of $78 \%$ between 1990 and 2010 [26]. Since the olive oil is in average less than $20 \%$ of the olive fruit, the increase of olive oil production has led to a proportional increase in olive mill by-products, comprising OVW [26]. The OVW possess beneficial properties for human health, including anti-inflammatory activity, and represent a huge source of useful compounds for many different biotechnological applications, including Cosmetics [27].

Recent studies proved the effects of hydroxytyrosol on inflammatory mediators, cytokines and chemokines, and identified anti-inflammatory constituents of aqueous olive extracts [28]. Aqueous olive extracts were fractionated by High Performance Liquid Chromatography (HPLC) and the fractions investigated for their effects on the production of inflammatory mediators nitric oxide (NO) and prostaglandin E2 (PGE2). It was established that hydroxytyrosol inhibited the production of NO and PGE2, reflecting strong anti-inflammatory activity and together with OVW diminished secretion of 
cytokines (IL-1 $\alpha$, IL-1 $\beta$, IL-6, IL-12, TNF- $\alpha$ ), and chemokines (CXCL10/IP-10, CCL2/MCP-1) [28]. Moreover, the beneficial properties of two fractions of OVW from Olea europea, Carolea variety, in combination with acacia fibers, were investigated. It was monitored a strong anti-inflammatory effect, antioxidant activity and detoxifying effect on cultured skin cells, which indicated that the fractions obtained from OVW could be developed as products for skin care [29].

\section{Valorization of By-Products Deriving from Pineapple and Grapevine}

As already mentioned, nearly all the by-products and their derivatives have some valuable applications, thus recently much effort has been concentrated on how to get even higher value from them in terms of efficacy [6]. Examples of this kind of valorization are the cases of pineapple rind and core by-products and the grape mark extracts.

Pineapple (Ananas comosus) is the most processed tropical fruit generating high quantity of by-products. These by-products represent $25 \%-35 \%$ of the pineapple fruit, being the rind and the core the predominant ones [30]. Pineapple rind and core have a high content of compounds with antioxidant activity, which give them a huge potential concerning their valorization in the cosmetics industry [31]. By using ultra-high performance liquid chromatographic (UHPLC) method, it was determined the presence of eight different carotenoids ( $\alpha$-carotene, $\beta$-carotene, $\beta$-cryptoxanthin, lutein, lycopene, neoxanthin, violaxanthin and zeaxanthin) and two vitamins (A and $\mathrm{E}$ ) in the pineapple rind and core, and the effect of Ultraviolet $\mathrm{C}(\mathrm{UV}-\mathrm{C})$ radiations on these compounds was evaluated [32].

The ultra-violet radiation at a wavelength of 190-280 nm (UV-C) is one of the main sustainable sanitation techniques of fruits, it is an environment friendly technique, relatively inexpensive. It also induces stress in the plant cells, can promote the biosynthesis of carotenoids and vitamin $\mathrm{C}$, and could give an added value to the by-products [33]. On this basis, it was confirmed that the level of L-ascorbic acid in the pineapple rind was higher compared with its level in the whole fruit and it was shown that UV-C radiation increased the L-ascorbic acid content in the rind. It was also established that, among the carotenoids, the most abundant was $\beta$-carotene, the carotenoid with the highest pro-vitamin A activity, whose concentration also increased in the rind after UV-C radiation exposure [32].

Grapevine is another example of species with high nutritional value, rich of beneficial compounds like vitamins, tannins, polyphenols and carotenoids. Around $15 \%$ of the crushed grapes become grape marc or pomace, the solid by-product left over from the winemaking process, which consists of pulp, skins and seeds [34]. Grape marc represents an industrial waste product which is utilized in other biotechnological processes, as the production of the Italian "Grappa", distillation of ethanol and tartaric acid extraction, purification of colorants and preparation of soil fertilizers [35]. The grape marc deriving from white wine production obtained from Aglianico grapes (the most valuable and the most prestigious grape variety in Campania region, Italy) is even richer in nutrients, since it is unfermented and no compound has yet been extracted by the wine making process. Moreover, it still contains several types of lipophilic compounds, such as resveratrol, lycopene, ellagic acid and especially carotenoids, which do not dissolve in the water soluble juice at the moment of pressing, but could be extracted by an oil-soluble (liposoluble) solvent and the obtained extract can be used as active ingredient for skin care Cosmetics [36]. 
On the basis of this assumption, a new cosmetic ingredient composed of a liposoluble fraction of the unfermented Aglianico marc, used for making white wine DOC (Controlled Designation of Origin), Sant' Agata dei Goti, Benevento, Italy, was developed. The liposoluble marc extract (LME) was mixed with another liposoluble extract obtained from Vitis vinifera cells (Aglianico cultivar), grown as sterile liquid cultures (liposoluble cell extract, LCE) and the obtained mixture was tested on cultured skin cells. It was studied the effect of the combination of the LME and LCE, mixed in a 10:1 ratio on proteasome activity in Human Dermal Fibroblasts (HDF) and human keratinocytes (HaCaT) [35]. "Proteasome" is the proteolitic system involved in the elimination of abnormal and damaged proteins and in the stressed cells its activity is inhibited by the generation of highly oxidized and aggregated proteins in the cytoplasm [37]. The tested mixture of LME and LCE gave strong effects in increasing the detoxifying capacity of the cells by up regulating the proteasome activity by $44 \%$ in fibroblasts and $61 \%$ in keratinocytes. Investigators have also proven the role of the LME/LCE mixture in stimulating new collagen production by measuring the expression level of Collagen I and Collagen III and in boosting the hydrating potential by measuring the expression level of HAS 2 (Hyaluronan Synthase 2) and APQ3 (Aquaporin 3) genes [35].

\section{Coffee Processing By-Products}

Another source of large amounts of disposable by-products is the coffee processing industry. Coffee plants (Coffea Arabica and Coffea robusta) are cultivated in many world countries, in particular those localized in the equatorial Latin America, South Asia, India and Africa. Coffee dispensation requires an elevated degree of processing know how and produces by-products such as coffee pulp and husk, which have limited applications as fertilizer, livestock feed and compost [38]. On the other hand, the manual step of coffee berry collection and selection can generate quantities of unused green beans, because they have been mechanically damaged or not sufficiently ripe and big to pass to the next processing step. These green beans are still a precious source of natural antioxidants, which have not yet been broken down by the roasting process [38].

Therefore, in order to have a valuable ingredient, rich in bioactive anti-oxidant compounds, unroasted coffee beans were collected, subjected to decaffeination using two natural elements, pure water and carbon dioxide $\left(\mathrm{CO}_{2}\right)$, and extracted in ethanol. After ethanol removal, it was observed that the jelly-like extract improved natural skin cell renewal, promoted an even skin tone for a radiant complexion and reinforced the epidermal barrier. Moreover, it helped prevent water loss, lighten the skin by inhibiting melanin synthesis and accelerated damaged skin repair, by decreasing the inflammatory process [39].

\section{Conclusions}

Sustainability is not just a new "buzzword" but it is indeed the major challenge facing humanity in the 21 st century as, according to the most recent United Nations forecast, the current world population of 7.2 billion is projected to increase by 1 billion over the next 12 years and reach 9.6 billion by 2050 [40]. Every sector of human activity will have to become sustainable. Agriculture, because it uses enormous land and water resources and contributes substantially to pollution, has the most serious sustainability issues and needs to change its practices. Besides contributing to sustainable development 
by supplying safer and more eco-friendly pest control systems, the agronomical industry may take more advantage of the huge amounts of by-products that derive from its activities and find a way to recycle with the minimum environmental impact. This means that the agricultural food industry should not only guarantee the final product with optimal characteristics (structure, nutritional value, safety, quality), but also should provide strategies to combine food production with minimization of water and energy use and proper valorization of wastes and by-products.

The cosmetic industry can certainly represent a remunerable solution on how recycling disposable by-products, since sustainability and human safety are also the most relevant issues for all the cosmetic products. Moreover, the cosmetic market is always growing, and despite the world economic crisis of the last years, over 5 billion cosmetic products are still sold every year, with the most consumed items being those for skin care.

In this review, we presented some example of food by-products extracts characterized for their cosmetic activity or already employed in cosmetic formulations, but many others could be tested and proposed for further applications, where less effective and more expensive products are currently being used.

Undoubtedly, extracts obtained from the by-products (those deriving from fruit and vegetable processing industries) represent a valid "green" alternative to the regular plant derived extracts, commonly used in cosmetics. Besides their proven beneficial effects on human skin, the agro-food by-products have the advantages of being bio-sustainable and inexpensive.

Concerning the safety issue, the food by-products have a complex composition of nutrients; thus, they are easily subjected to microbial contamination and may sometimes contain pesticide residues if the regulations of biological farming have not fully respected by the producers. In those cases it would be necessary to use specific and sensible analyses to detect the presence of any type of anomalies in a certain agro-food by-product, prior to its characterization and valorization as active ingredient for Cosmetics. Another consideration is that despite of the proven qualities of the food by-products, there could be a certain variability in the composition due to their seasonal production. Thus, it becomes necessary to choose the right industries that work in the total respect of the Good manufacturing practices (GMP) regulations and make all the possible efforts to standardize the production processes, in order to have always the same characteristics of quality from one batch of by-product to the other.

\section{Conflicts of Interest}

The authors declare no conflict of interest.

\section{Author Contributions}

All the authors contributed to the writing and editing of the article.

\section{References}

1. Barbulova, A.; Apone, F.; Colucci, G. Plant cell cultures as source of cosmetic active ingredients. Cosmetics 2014, 2, 94-104. 
2. Oksman-Caldentey, K.M.; Inze, D. Plant cell factories in the post-genomic era: New ways to produce designer secondary metabolites. Trends Plant Sci. 2004, 9, 433-440.

3. Djilas, S.; Canadanovic-Brunet, J.; Cetkovic, G. By-products of fruits processing as a source of phytochemicals. Chem. Ind. Chem. Eng. Q. 2009, 15, 191-202.

4. Kris-Etherton, P.M.; Lefevre, M.; Beecher, G.R.; Gross, M.D.; Keen, C.L.; Etherton, T.D. Bioactive compounds in nutrition and health-research methodologies for establishing biological function: The antioxidant and anti-inflammatory effects of flavonoids on atherosclerosis. Annu. Rev. Nutr. 2004, 24, 511-538.

5. Ayala-Zavala, J.; Rosas-Domínguez, C.; Vega-Vega, V.; González-Aguilar, G.A. Antioxidant enrichment and antimicrobial protection of fresh-cut fruits using their own byproducts: Looking for integral exploitation. J. Food Sci. 2010, 75, R175-R181.

6. Tuck, C.; Perez, E.; Horvath, I.; Sheldon, R.; Poliakoff, M. Valorization of biomass: Deriving more value from waste. Science 2012, 337, 695-699.

7. Goñi, I.; Hervert-Hernández, D. By-Products from Plant Foods are Sources of Dietary Fibre and Antioxidants, Phytochemicals-Bioactivities and Impact on Health, 2011. Available online: http://www.intechopen.com/books/phytochemicals-bioactivities-and-impacton-health/by-productsfrom-plant-foods-are-sources-of-dietary-fibre-and-antioxidants (accessed on 2 December 2014).

8. Schieber, A.; Stintzing, F.; Carle, R. By-products of plant food processing as a sour02ce of functional compounds-recent developments. Trends Food Sci. Technol. 2001, 11, 401-413.

9. Liu, Y.Q.; Heying, E.; Tanumihardjo, S.A. History, global distribution, and nutritional importance of citrus fruits. Compr. Rev. Food Sci. Food Saf. 2012, 11, 530-545.

10. Kim, S.; Kim, M.J.; Choi, Y.; Kim, B.; Kim, K.; Park, K.; Park, S.; Lee, N.; Hyun, N. Down-regulation of tyrosinase, TRP-1, TRP-2 and MITF expressions by citrus press-cakes in murine B16 F10 melanoma. Asian Pac. J. Trop. Biomed. 2013, 3, 617-622.

11. Kim, S.S.; Lee, J.A.; Kim, J.Y.; Lee, N.H.; Hyun, C.G. Citrus peel wastes as functional materials for cosmeceuticals. J. Appl. Biol. Chem. 2008, 51, 7-12.

12. Balkrishnan, R.; McMichael, A.J.; Hu, J.Y.; Camacho, F.T.; Shew, K.R.; Bouloc, A.; Rapp, S.R.; Feldman, S.R. Correlates of health-related quality of life in women with severe facial blemishes. Int. J. Dermatol. 2006, 45, 111-115.

13. Costin, G.E.; Hearing, V.J. Human skin pigmentation: Melanocytes modulate skin color in response to stress. FASEB J. 2007, 21, 976-994.

14. Yen, F.L.; Wang, M.C.; Liang, C.; Ko, H.H.; Lee, C.W. Melanogenesis inhibitor(s) from Phyla nodiflora extract. Evid. Based Complement. Alternat. Med. 2012, 2012, doi:10.1155/2012/867494.

15. Pavithra, P.S.; Sreevidya, N.; Verma, R.S. Antibacterial activity and chemical composition of essential oil of Pamburus missionis. J. Ethnopharmacol. 2009, 124, 151-153.

16. Baik, J.S.; Kim, S.S.; Lee, J.L.; Oh, T.H.; Kim, J.Y.; Lee, N.H.; Hyun, C.G. Chemical composition and biological activities of essential oils isolated from Korean endemic Citrus species. J. Microbiol. Biotechnol. 2008, 18, 74-79.

17. Yang, E.J.; Kim, S.S.; Oh, T.H.; Baik, J.S.; Lee, N.H.; Hyun, C.G. Essential oil of citrus fruit waste attenuates LPS-induced nitric oxide production and inhibits the growth of skin pathogens. Int. J. Agric. Biol. 2009, 11, 791-794. 
18. Muracami, A. Chemoprevention with phytochemicals targeting inducible nitric oxide synthase. Forum Nutr. 2009, 61, 193-203.

19. Available online at Tomato Processing Industries. Available online: http://www.tomatonews.com/ resources.html (accessed on 2 December 2014).

20. Borguini, R.G.; Torres, E.A. Tomatoes and tomato products as dietary sources of antioxidants. Food Rev. Int. 2009, 25, 313-325.

21. Papaioannou, E.; Karabelas, A. Lycopene recovery from tomato peel under mild conditions assisted by enzymatic pre-treatment and non-ionic surfactants. Acta Biochim. Polonica 2012, 59, 71-74.

22. Zuorro, A.; Lavecchia, R. Mild enzymatic method for the extraction of lycopene from tomato paste. Biotechnol. Biotechnol. Equip. 2010, 24, 1854-1857.

23. Cicerale, S.; Conlan, X.A.; Sinclair, A.J.; Keast, R.S. Chemistry and health of olive oil phenolics. Crit. Rev. Food Sci. Nutr. 2009, 49, 218-236.

24. Omar, S.H. Oleuropein in olive and its pharmacological effects. Sci. Pharm. 2010, 78, 133-154.

25. Visioli, F.; Galli, C. Biological properties of olive oil phytochemicals. Crit. Rev. Food Sci. Nutr. 2002, 42, 209-221.

26. International Olive Council, World Olive Figures, 2011. Available online: http://www. internationaloliveoil.org/estaticos/view/131-world-olive-oil-figures (accessed on 2 December 2014).

27. Bitler, C.M.; Viale, T.M.; Damaj, B.; Crea, R. Hydrolyzed olive vegetation water in mice has anti-inflammatory activity. J. Nutr. 2005, 135, 1475-1479.

28. Richard, N.; Arnold, S.; Hoeller, U.; Kilpert, K.; Wertz, K.; Schwager, J. Hydroxytyrosol is the major anti-inflammatory compound in aqueous olive extracts and impairs cytokine and chemokine production in macrophages. Plant. Med. 2011, 77, 1890-1897.

29. Sustainable Olive Derivative to Shield Cells from Oxidative and Inflammatory Damages. Available online: http://www.vitalabactive.com/actives/pureolea-vita.html (accessed on 2 December 2014).

30. Reinhard, A.; Rodriguez, V.L. Industrial processing of pineapple-Trends and perspectives. Acta Hortic. 2009, 822, 323-328.

31. Wu, Z.; Zhang, M.; Wang, S. Effects of high-pressure argon and nitrogen treatments on respiration, browning and antioxidant potential of minimally processed pineapples during shelf life. J. Sci. Food Agric. 2012, 92, 2250-2259.

32. Freitas, A.; Moldao-Martins, M.; Costa, H.; Albuquerque, T.; Valentea, A.; Sanches-Silvaa, A. Effect of UV-C radiation on bioactive compounds of pineapple (Ananas. comosus L. Merr.) by-products. J. Sci. Food Agric. 2015, 95, 44-52.

33. Gonzalez-Aguilar, G.A.; Villa-Rodriguez, J.A.; Ayala-Zavala, J.F.; Yahia, E.M. Improvement of the antioxidant status of tropical fruits as a secondary response to some postharvest treatments. Trends Food. Sci. Technol. 2010, 21, 475-482.

34. Caimari, A.; del Bas, J.M.; Crescenti, A.; Arola, L. Low doses of grape seed procyanidins reduce adiposity and improve the plasma lipid profile in hamsters. Int. J. Obes. 2013, 37, 576-583.

35. Carola, A.; Tito, A.; Bimonte, M.; Mustilli, A.; Cucchiara, M.; Monoli, I.; Hill, J.; Apone, F.; Colucci, G. Liposoluble extracts of Vitis vinifera grape marc and cell cultures with synergistic anti-ageing effects. HPC Today 2012, 7, 42-46.

36. Oliveira, C.; Ferreira, A.C.; Costa, P.; Guerra, J.; Guedes de Pinho, P. Effect of some viticultural parameters on the grape carotenoid profile. J. Agric. Food Chem. 2004, 52, 4178-4784. 
37. Bulteau, A.L.; Moreau, M.; Nizard, C.; Friguet, B. Impairment of proteasome function upon UVA- and UVB-irradiation of human keratinocytes. Free Radic. Biol. Med. 2002, 32, 1157-1170.

38. Murthy, S.; Naidu, M. Sustainable management of coffee industry by-products and value addition-A review. Resour. Conserv. Recycl. 2012, 66, 45-58.

39. A Sustainable Natural Product from Decaffeinated Green Coffee Beans to Achieve a Smooth and Radiant Skin. Available online: http://www.vitalabactive.com/actives/green-coffea-vita.html (accessed on 2 March 2015).

40. World Population Prospects: The 2012 Revision; United Nations Department of Economic and Social Affairs, Population Division: New York, NY, USA, 2013.

(C) 2015 by the authors; licensee MDPI, Basel, Switzerland. This article is an open access article distributed under the terms and conditions of the Creative Commons Attribution license (http://creativecommons.org/licenses/by/4.0/). 